\title{
On-line Monitoring of the Laser Joining Process of Aluminum Alloy and Polyamide 6.6 by Acoustic Emission Analysis
}

\author{
By Peter Boehm* \\ Christian Lamberti ${ }^{\dagger}$
}

A novel Laser joining process for polyamide and aluminium hybrid structures has proven a strong bond between these dissimilar materials. This newly developed welding technique is strongly requested by the automobile producing industry for several applications within the car structure. However, the joining quality exhibits a strong dependency to process-related variables, which highlights the need for an online inspection technique. In that case only the acoustic emission analysis (AEA) as a Non-destructive Technique (NDT) can be used to guarantee reasonable results. Sometimes the break of the melting bath can occur and is often not recognizable by optical methods. The AEA however was able to detect this lack of fusion. The appearance of gas bubbles, arising from the PA 6.6 could be detected by the AEA. The signals related to this effect can be correlated clearly to micrographic sections. Future tasks will develop algorithms to separate the different defects by pattern recognition of the AEA signals and parameters.

Keywords: Acoustic Emission Analysis, Aluminium alloy, Laser joining of metals and plastics, Polyamide 6.6

\section{Introduction}

Plastics place their important role within the bandwidth of lightweight construction materials because of their marginal mass density of about $1 \mathrm{~g} / \mathrm{cm}^{3}$ in comparison to steel $\left(7.8 \mathrm{~g} / \mathrm{cm}^{3}\right)$. This property helps the plastics to find more and more fields of application within the automobile industry, air craft industry as well as in the plant engineering. The weight percentage of plastics within the automobile industry increases from $10 \%$ in 2004 up to $20 \%$ in the year of 2014. New constructed air crafts like the Airbus A 380 insert more often reinforced plastics, arising from the demand to lower costs, reduced weight and in many cases because of more flexible production qualities. On the other hand some complex structures cannot be produced completely from plastic materials, as plastics do not possess the required stiffness and stability qualities. To find a remedy a combination of plastics with metals to a hybrid product can help. Hybrid material combinations like Aluminium with a

\footnotetext{
* Associate Professor, University of Applied Sciences Trier, Germany.

${ }^{\dagger}$ PhD Student, University of Luxembourg, Luxembourg.
} 
thermoplastic material have to be joined to create a stable compound. Up to now combinations of Metal/plastic had been glued besides the mechanical attachment by screws or rivets. Adhesive bonds are often not resistant to age and are strongly influenced by temperature, sun light and/or stress. Therefore joining methods are welcome to replace adhesive bonds and to reach at least their joining qualities. The welding of metals, as well as the welding of plastics, is well known over a long time period - but - only within their own material category. A welding process to join both material categories has not been reached up to now. The big problem corresponds to the great difference due to their melting points. The melting point of Aluminium lies within a region in which plastics are normally thermally degraded (Amend et al., 2013; Jung et al., 2013a). A newly developed method to join both materials is researched recently at the University of Luxembourg (Lamberti et al., 2014). The energy for the welding process is realized by a special type of Laser. By focusing optics like mirrors or lenses the Laser beam is bundled. Throughout the focusing technique the Laser beam reaches the required beam intensity to fuse the metal surface. The Laser beam gets absorbed and the material gathers energy to fuse finally. During the process the Laser moves with a specific feed rate over the Aluminium surface and produces a joining seam. On the basis of the reflection of the Laser beam metals absorb only a low part of radiation energy (steel about $5 \%$ ). Aluminium has higher grade of reflection because of its oxide layer. Due to the high intensity of the beam the material will be vaporized and ionized. A vapor channel occurs, possessing the same diameter like the focused Laser beam. The metal vapor which flows off enables an increased absorption of the Laser beam. The energy coming out of the metal vapor is transferred to the material. This fact leads to deeper penetration of the Laser beam and to a further vaporization effect. Moreover the Laser beam is reflected several times at the capillary wall and increases the effectivity. The appearance of the vapor channel allows the effect of a deep welding zone. The vapor channel is surrounded during the welding process by the Aluminium molten mass. The vapor pressure prevents the collapse of the vapor capillary (Farazila et al., 2012; Jung et al., 2013b).

To check the quality of the produced weld seam at time only a visual inspection of the surface or the destruction of the welded component and an examination helped by metallografic methods take place, Figure 1 . If this new developed welding method would find its way out of the laboratory stadium a non-destructive method is in demand, which can give direct information about the quality of the weld seam during the welding process.

A suchlike method is the so called Acoustic Emission Analysis (AEA) (Ismail et al., 2013; Crostack et al., 1991). This method is used amongst others at the University of Applied Sciences in Trier. To find out if this testing method could provide reliable results both Universities work together in some projects. Acoustic emissions are sound waves which are produced in a solid body for example under mechanical stress.

The AEA is applied in many fields of industry. It is used for instance to detect cracks in the work piece during the production process or the on-line 
monitoring of tanks, gas spheres and more and more to monitor the pressure test of high pressurized vessels (Böhm, 1991). The AEA operates according to the principle - detection of acoustic emissions triggered by material actions. For the detection of the sound waves acoustic sensors, comparable to ultrasonic sensors, are used. The difference is - they are only working in a passive way. The detected signals are given to an AEA system which includes preamplifiers, signal filtering units and the analysis unit. The main goal of our experiments lies within the question - are we able to detect and to analyze the sound waves emitted by the welding process and are we able to allocate the analyzed data to the occurred material events during the welding process? Moreover it is of great interest to correlate the analyzed data to the quality of the welded material.

Figure 1. Cross Sectional Micrograph of the Weld Seam Aluminium/Polyamide 6.6

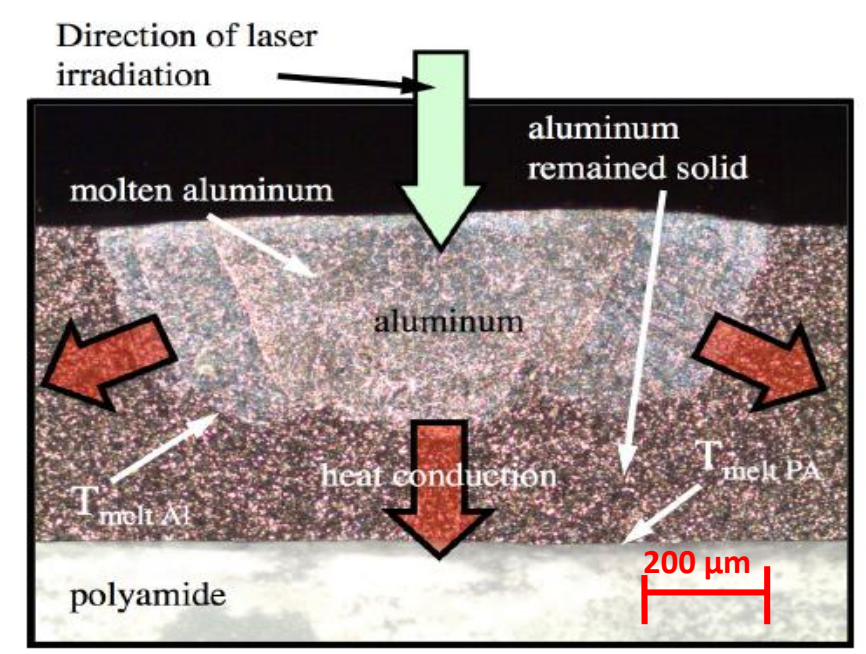

\section{Experimental Setup}

Initially it was examined if the modulation frequency of the Laser beam has effects on the AE-signals. After some tests it could be stated that the spatial as well as the temporal modulation frequency creates an influence on the signals. The Laser was pulsed by a frequency of $10 \mathrm{kHz}$ and with a pulse length of $10 \mu \mathrm{s}$. The maximum Laser power was about $380 \mathrm{~W}$, the spatial modulation frequency represents $500 \mathrm{~Hz}$. The analog signal was digitized by a sampling rate of $1 \mathrm{MHz}$. The shifting of the Laser beam is schematically shown in Figure 2. The Laser beam moves forward in ellipsoid, overlapping trails. 
Figure 2. Spatial Power Modulation by Superposed Beam Oscillation on the Linear Feed

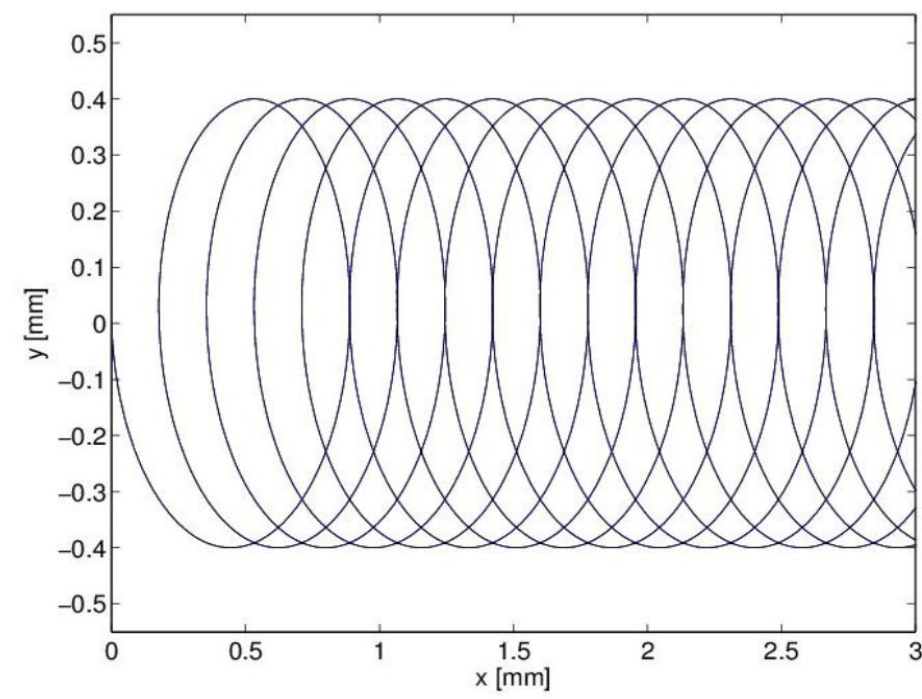

For the detection of the AE signals a multi-channel system produced by Vallen Systeme GmbH was used. It has the ability to digitize and to store the time signals. Moreover it allows a frequency analyzation and selects important signal parameters on-line to show them in elected diagrams. During the welding process a lot of AE-Signals had been detected and analysed, Figure 3. The left partial image shows the temporal modulation frequency by the pulse energy, the right partial image gives the result of the spatial modulation frequency of the Laser. At this particular time of the impulse $(0.186 \mu \mathrm{s})$ the Laser finds itself again at the initial point, standing in an area which was already moved over by the Laser. This type of movement clarifies the difference in pulse intensity at the temporal modulation frequency. If the Laser pulse meets already molten Aluminium, a higher AE-pulse is generated. Therefore, it was clear that the Laser welding process initiates actual sound signals which correlate exactly with the modulation frequency of the Laser beam. Up till now it has been found how far the sound emitting material activities can be correlated to the AEA (Böhm et al., 2015).

Figure 3a. Temporal Modulation Frequency of the Laser
Figure 3b. Spatial Modulation Frequency of the Laser

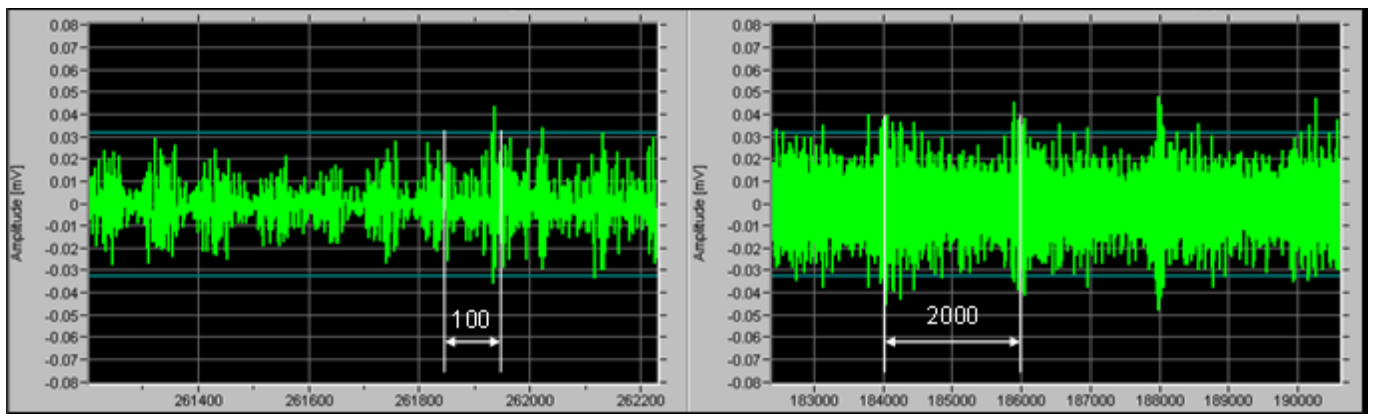




\section{Consequence of the Laser Power on the Acoustic Emission}

To understand how far the Laser power influences the quality of the weld seam the Laser power was verified and correlated with the AE-signals in further tests. For further experiments we selected 5 power levels of $220 \mathrm{~W}, 240$ $\mathrm{W}, 320 \mathrm{~W}$ and $350 \mathrm{~W}$. The Laser moved forward with a constant velocity of 50 $\mathrm{mm} / \mathrm{s}$. The sound signals, detected over an estimated trigger level were transformed on-line in a so called amplitude-time diagram. In Figure 4 the influence of the increasing Laser power shows an explicit correlation. More Laser power creates higher AE-signals. This result was proven over all Laser power levels.

Figure 4. Amplitude-time Diagrams correlating to a Laser Power of $220 \mathrm{~W}$ and $280 \mathrm{~W}$

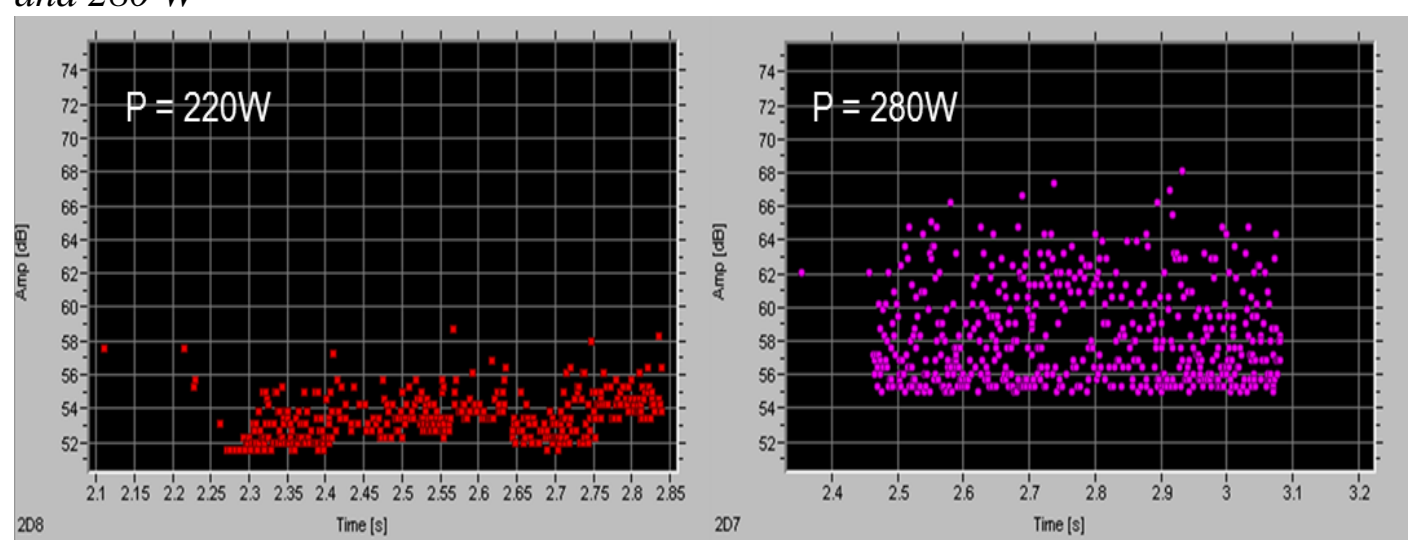

\section{Detection of AE Signals Showing the Tearing of the Melting Bath}

Using low Laser power levels (220-240 W) a tearing of the melting bath is presumably possible. Subsequently an insufficient link between the Aluminium and Polyamide surface can occur. Figure 5 shows an Aluminium weld correlated to the AE result as well as to the surface of the Polyamide after having destroyed the weld seam. In the beginning no satisfying link between the two materials can be registered, the melting bath breaks away in many stages. The AE detects some amplitude peaks. Only within the last third of the weld seam a high quality weld seam can be recorded. The AE-signal features higher and coherent amplitudes over a longer time period. So far it can be stated that the height, as well as the duration of the signals is coupled to the quality of the link between PA and Aluminium. 
Figure 5. Exposure of the Welding Result Correlated to AE Signals
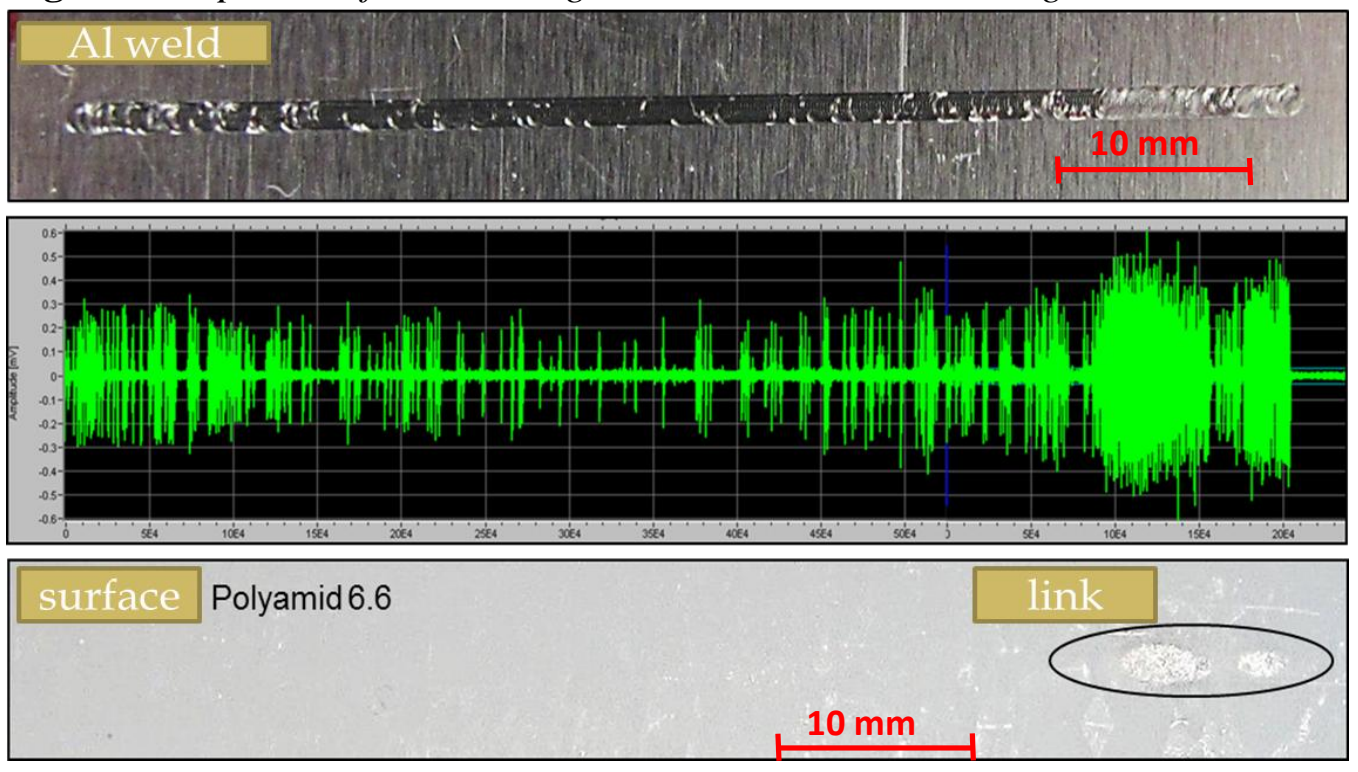

Figure 6. Correlation of AE Signals and the Formation of Gas Pores
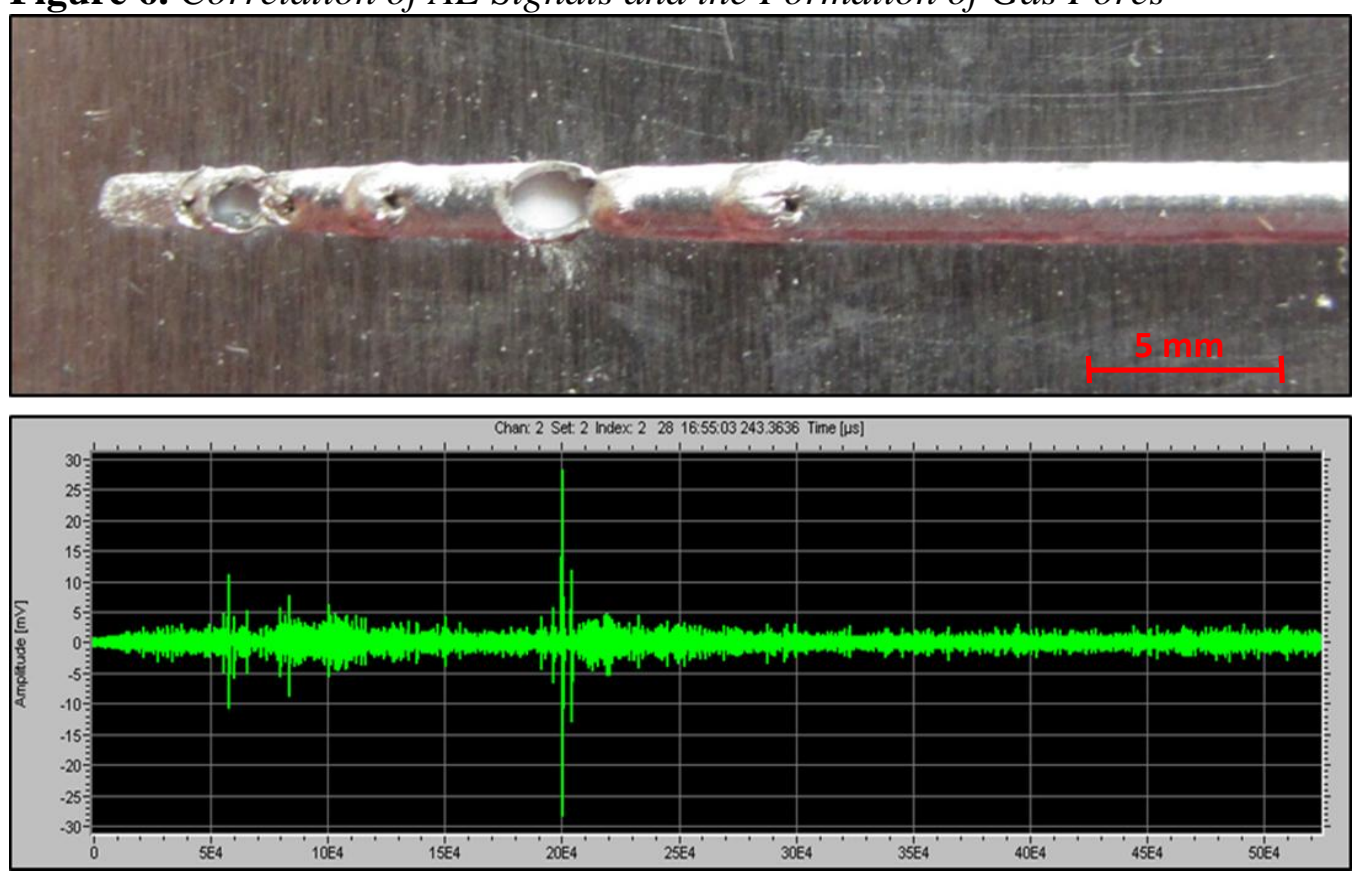

Other phenomenon, like the fracturing of the Aluminium weld by reason of an excessive vapour pressure caused by too high Laser power levels, can also be detected and evaluated, Figure 6. During this process some parts of the Aluminium melt are thrown out of the weld. The AE detects this fact by showing extremely high and single shots within the continuous signal sequence. Other methods of evaluation like the frequency analysis indicate in first tests that the spurt out of the molten metal parts can be separated in the frequency spectrum from other signals. 
The correlation between $\mathrm{AE}$ and the welding process describes in this stage of the experiments some different material behaviour. Some other material activities and their acoustic behaviour during the welding process are still unknown. So far it can be stated that the signals are strongly addicted to the height of the gas pressure and the mass of the thrown out particles. Explicit assignments of the AE signals to the material activities can in no case be warranted.

Figure 7. Correlation of AE Signals and the Quality of the Weld Seam
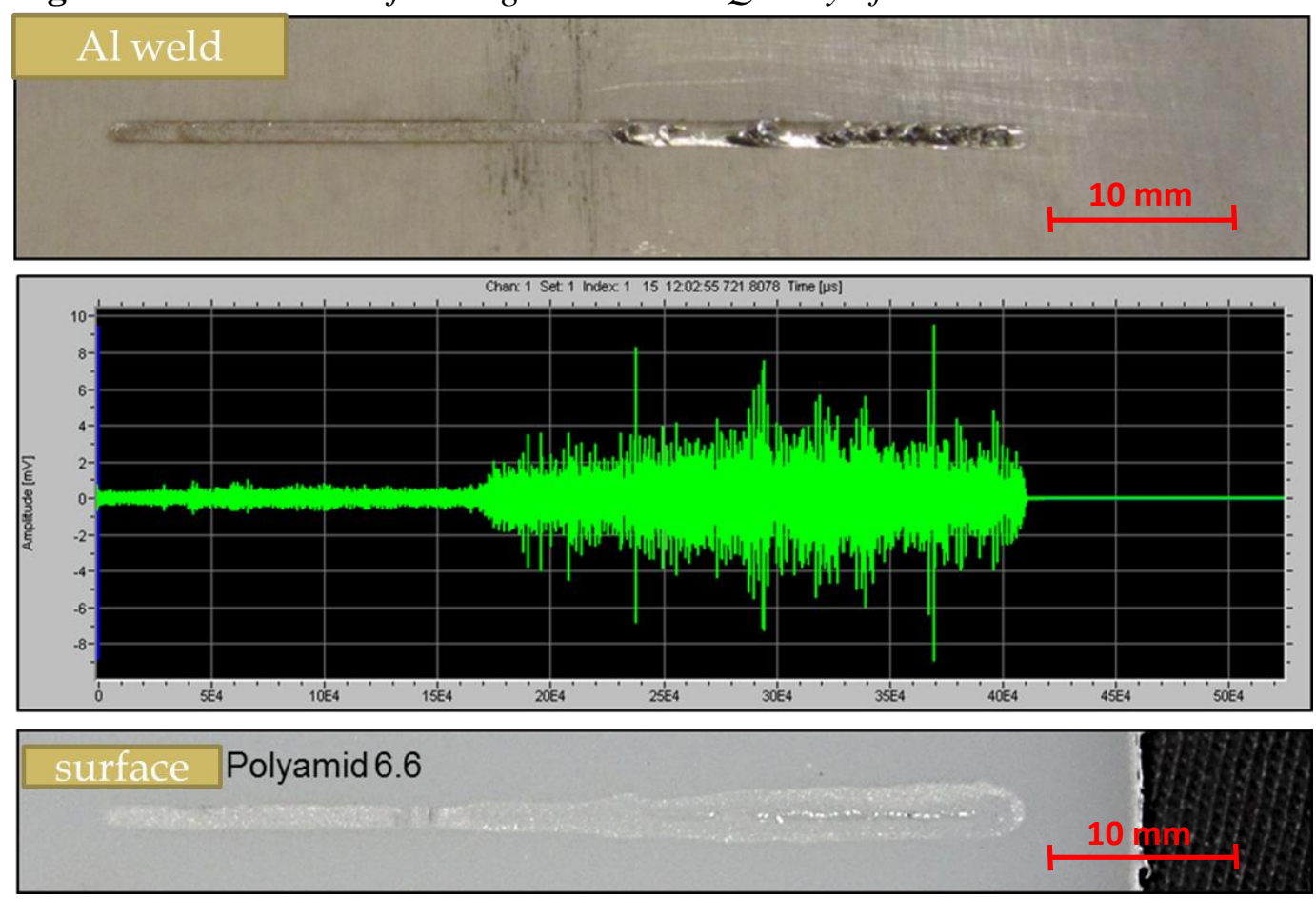

In Figure 7 a result is given, showing in the first half of the weld seam, relatively high quality. In the second half of the weld seam many gas pores arise, the thermoplastic material tends to combust. The AE signals react by rising up the height of the signals, followed by extreme high bursts, which can be correlated exactly to the extreme deep gas pores. Within the first half of the weld seam the AE gives a continuous signal trend within a limited range of signal height.

\section{Results and Discussion}

It can be stated that the Acoustic Emission Analysis has the ability to detect and to evaluate the material activities during the Laser welding process between Aluminium and Polyamide. Material activities like forming gas pores in the metal or low quality links to the Polyamide can be detected and allocated. Some welding parameters like Laser power and focus depth have been verified and correlated to the AE results. At this point of research some of 
the material activities are clearly identified. A clear correlation between all process parameters is still missing. Ongoing studies will cover the field of the clustering of the different $\mathrm{AE}$ sources and the definition of critical failures. More over the influence of the sensor positions has to be reduced or eliminated. An ultimate result has to be - on-line correlation of the AE signals to the welding process parameters to control the welding quality and to regulate the parameters within the batch manufacturing.

\section{Conclusions}

During the Laser welding process between Aluminium and Polyamide 66 the acoustic emission analyses method detects different sound signals which are emitted by occurred material activities. The signals are stored and evaluated by some mathematical algorithms. The laser activity itself, as well as some typical failures like gas pores, which can occur during the welding process, are registered and can be allocated to the different material activities. As a result, the acoustic emission system can differentiate between joins of good and minor quality. The next step will be the experimental examination of the influence of all parameters of the welding process by means of the AEA. Finally an automatic system should work as a monitoring control system to the whole welding process on-line in order to support the quality system of manufacturing lines.

\section{References}

Amend, P., Pfindel, S. and Schmidt, M. (2013) Thermal joining of thermoplastic metal hybrids by means of mono- and polychromatic radiation. Technical Report. Bayerisches Laserzentrum GmbH.

Böhm, P. (1991) Acoustic Emission Inspections of Nuclear Components Considering Recent Research Programmes, Charlotte, USA 20./23.03. 1989, Symposium: World Meeting on Acoustic Emission, Acoustic Emission-current practice and future directions, Sachse/Roget/Yamaguchi ASTM SPT 1077, ASTM pub. code (PCN) 04-010770-22, Baltimore, S. 78/83, 1991.

Böhm, P., Plapper, P., Lamberti, C. and Neumann, L. (2015) On-line monitoring des Laserstrahlprozesses von Aluminium mit einem thermoplastischen Kunststoff [On-line monitoring of the laser joining process of Aluminium to a thermoplastic resin]. Lehre und Forschung 2015 (im Druck), Hochschulreport der Hochschule Trier.

Crostack, H.A., Storp, H. and Böhm, P. (1991) Use of non-acoustic transducers for process control during electron beam welding. Charlotte, USA 20./23.03. 1989, Symposium: World Meeting on Acoustic Emission, Acoustic Emission-current practice and future directions, Sachse/Roget/Yamaguchi ASTM SPT 1077, ASTM pub. code (PCN) 04-010770-22, Baltimore, p. 35/46.

Farazila, Y., Miyashita, Y., Mutoh, Y. and Mohd Hamdi, A.S. (2012) Effect of anodizing on pulsed Nd:YAG laser joining of polyethylene terephthalate (PET) and aluminium alloy (A5052). Materials and Design, 37, p. 410-415. 
Ismail, Z. et al. (2013) Acoustic emission signals detected during the resistance spot welding of aluminum alloy. Measurement 46.3, p. 1053-1060.

Jung, K.W., Kawahito, Y., Takahashi, M. and Katayama S. (2013a) Laser direct joining of carbon fiber reinforced plastic to zinc-coated steel. Materials and Design, 47, p. 179-188.

Jung, K.W., Kawahito, Y., Takahashi, M. and Katayama S. (2013b) Laser direct joining of carbon fiber reinforced plastic to aluminum alloy. Journal of Laser Applications 25.3.

Lamberti, C., Solchenbach, T., Plapper, P. and Possart, W. (2014) Laser Assisted Joining of Hybrid Polyamide-Aluminum Structures. Physics Procedia, 56, p. 845 -853 . 
